\title{
Liposomes and nanotechnology in drug development: focus on neurological targets
}

This article was published in the following Dove Press journal:

International Journal of Nanomedicine

I March 2013

Number of times this article has been viewed

\section{Pedro Ramos-Cabrer Francisco Campos \\ Clinical Neurosciences Research Laboratory, Department of Neurology, Hospital Clínico Universitario de Santiago, University of Santiago de Compostela, Health Research Institute of Santiago, Santiago de Compostela, Spain}

Correspondence: Pedro Ramos-Cabrer Laboratorio de Neurociencias Clínicas, Hospital Clínico Universitario, Travesa da Choupana, 15706 Santiago de

Compostela, Spain

$\mathrm{Tel}+3498195 \quad 1097$

$\mathrm{Fax}+34981951098$

Email pedro.ramos.cabrer@sergas.es
Abstract: Neurological diseases represent a medical, social, and economic problem of paramount importance in developed countries. Although their etiology is generally known, developing therapeutic interventions for the central nervous system is challenging due to the impermeability of the blood-brain barrier. Thus, the fight against neurological diseases usually struggles "at the gates" of the brain. Flooding the bloodstream with drugs, where only a minor fraction reaches its target therapeutic site, is an inefficient, expensive, and dangerous procedure, because of the risk of side effects at nontargeted sites. Currently, advances in the field of nanotechnology have enabled development of a generation of multifunctional molecular platforms that are capable of transporting drugs across the blood-brain barrier, targeting specific cell types or functional states within the brain, releasing drugs in a controlled manner, and enabling visualization of processes in vivo using conventional imaging systems. The marriage between drug delivery and molecular imaging disciplines has resulted in a relatively new discipline, known as theranostics, which represents the basis of the concept of personalized medicine. In this study, we review the concepts of the blood-brain barrier and the strategies used to traverse/bypass it, the role of nanotechnology in theranostics, the wide range of nanoparticles (with emphasis on liposomes) that can be used as stealth drug carriers, imaging probes and targeting devices for the treatment of neurological diseases, and the targets and targeting strategies envisaged in the treatment of different types of brain pathology.

Keywords: nanotechnology, theranostics, blood-brain barrier, brain, central nervous system

\section{The CNS and blood-brain barrier:"the enemy at the gates"}

Neurological diseases represent a medical, social, and economic problem of paramount importance in developed countries, in particular because their incidence is increasing rapidly with the progressive rise in life expectancy. ${ }^{1}$

Although the etiology of most neurological diseases is known and experimental studies have continuously provided potential drugs for their treatment, the performance of therapeutic interventions in the central nervous system (CNS) remains a challenge. The CNS is a complex and vulnerable system, and its evolution has provided it with effective mechanisms of defense against foreign elements. Ironically, the strength of these defensive mechanisms usually complicates attempts to perform therapeutic interventions within the CNS. ${ }^{2}$ Thus, the fight against neurological diseases usually struggles "at the gates" of the brain.

There are three main barriers that regulate molecular exchange between the blood and brain parenchyma, including the blood-brain barrier, which is formed by the interaction 
between glial cells and endothelial cells of the blood vessels in the brain, the choroid plexus epithelium, which is the border between the blood and ventricular cerebrospinal fluid, and the arachnoid epithelium, which separates the blood from the subarachnoid cerebrospinal fluid.

The blood-brain barrier represents the main gateway via which systemically administered drugs access the brain and is the structural basis of the functional system known as the neurovascular unit, which is formed by a monolayer of endothelial cells connected to each other via tight junctions, as well as by astrocytic end feet, perivascular neurons, and pericytes (Figure 1). ${ }^{3,4}$ Endothelial cells of the brain are highly polarized and show low pinocytic activity, but contain different active transport mechanisms to ensure homeostasis of the brain. Endothelial cells also contain large concentrations of P-glycoprotein. P-glycoprotein is an ATP-dependent protein that actively transports a wide range of drugs out of the brain. P-glycoprotein inhibits penetration of relatively large $(>400 \mathrm{Da})$ hydrophobic drugs into the brain via active back transport of these drugs into the blood. ${ }^{5}$

Thus, the blood-brain barrier is permeable to small and lipophilic molecules (eg, oxygen, carbon dioxide, and nicotine) and larger molecules which do not spontaneously diffuse across it and must be transported across the bloodbrain barrier via specific carrier systems (ie, systems used for transport of glucose and essential amino acids) or by receptormediated endocytosis systems (eg, systems used for leptin, transferrin, or insulin) which are present in the membrane of the endothelial cell (Figure 1). For those molecules that cannot make use of one of the aforementioned transport mechanisms, different strategies can be used to transport these molecules into the brain parenchyma to reach the site of pathology.

One way to circumvent the blood-brain barrier is direct administration of an intraparenchymal injection of the desired substance. Another is to use implantable systems, such as osmotic pumps or enhanced convention devices, for continuous drug infusion. However, surgical approaches are invasive, risky, and cannot be used when the implantable device crosses or targets critical areas of the brain. It is also possible to use such devices to perform intraventricular or intrathecal administration of drugs when involving the cerebrospinal fluid-brain barrier to avoid the blood-brain barrier. Intranasal delivery is also an emerging noninvasive alternative to bypass the blood-brain barrier and facilitates delivery of large charged therapeutic molecules into the brain. $^{7}$

Rather than bypassing the blood-brain barrier, it is possible to disrupt it temporarily by opening the tight junctions of endothelial cells. Under these conditions, permeability of the blood vessels in the brain to systemically applied treatments can be increased. ${ }^{8}$ A transient disruption of the blood-brain barrier may be achieved by: osmotic shock using mannitol, arabinose, or other hypertonic solutions; acting at specific receptors on endothelial cells with substrates that affect the tight junctions, such as bradykinin receptors; ${ }^{9}$ and using magnetic resonance imaging-guided ultrasound in combination with microbubbles of contrast agents to induce focalized openings in small areas of the brain. ${ }^{10}$ Opening of the blood-brain barrier may be a dangerous procedure because it can cause hydroelectrolytic changes and suppress the mechanisms that regulate the entrance of substances into the brain. ${ }^{11,12}$
A

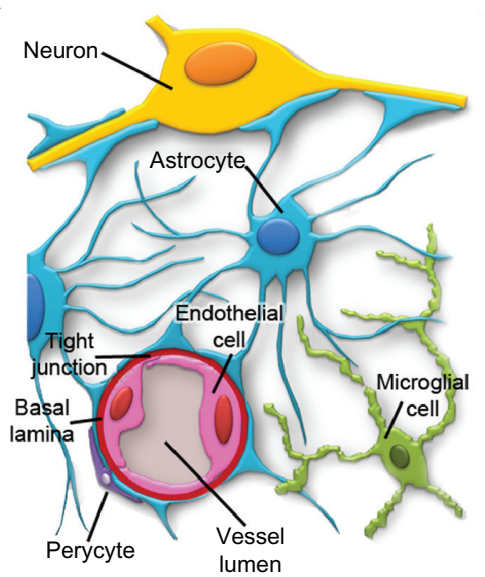

B

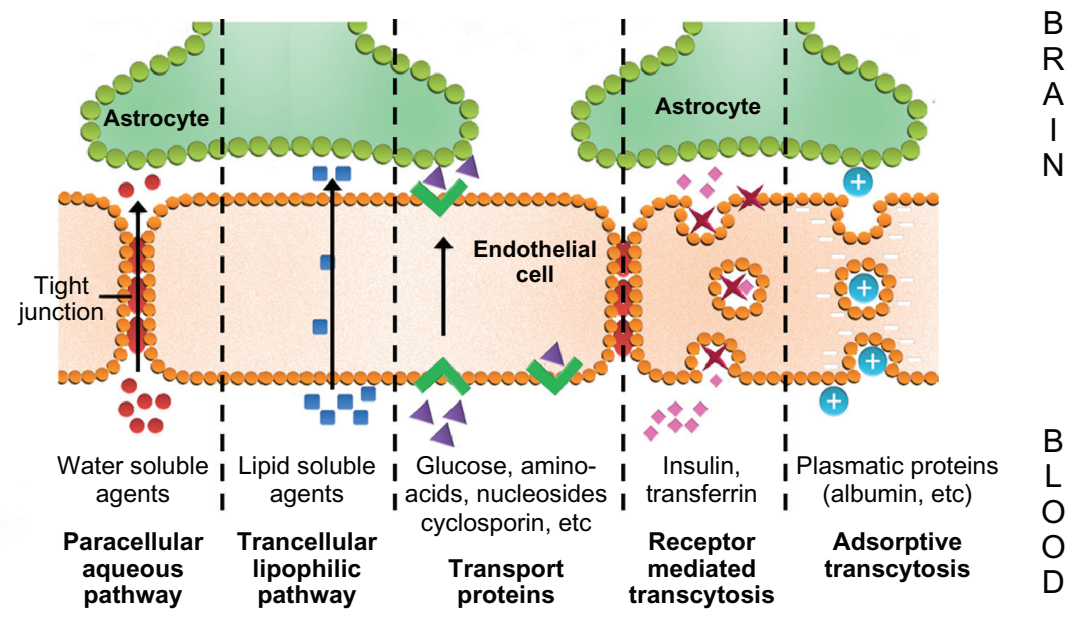

Figure I (A) Structure of the blood-brain barrier, established by the endothelial cells of blood capillaries and their tight junctions (B) Different mechanisms for the transporting of substances across the blood-brain barrier. Adapted from Mol Med Today, 2, Abbott NJ, Romero IA, Transporting therapeutics across the blood-brain barrier. 106-1 13. Copyright (1996), with permission from Elsevier.6 
There are alternative methods for distributing drugs inside the brain parenchyma without altering the blood-brain barrier when the aforementioned transport mechanisms are not suitable or unavailable. The first option is to use chemical derivatives or to partially modify the structure of the drug to facilitate traversing of the blood-brain barrier while maintaining drug activity (eg, by adding aliphatic chains to increase lipophilicity). ${ }^{13}$ A second possibility is to use prodrugs, ie, reversible compounds that in their native state are not biologically active, but are able to cross the blood-brain barrier and undergo an enzymatic or chemical transformation once in the brain parenchyma, thereby becoming active. ${ }^{14}$

The most versatile and attractive approach for delivery of drugs in their native state into the brain parenchyma involves use of drug carriers as "Trojan horses", ie, nanoscaled molecular platforms carrying therapeutic compounds that can cross the blood-brain barrier. In this context, nanotechnology represents a key component in the development of effective treatments for diseases of the CNS.

Multiple structures have been proposed for drug delivery in recent years. In general, there are two large families of transporters, ie, reversible and irreversible nanoparticles. Reversible nanoparticles are supramolecular complexes generated on the basis of noncovalent intermolecular interactions, ie, Van der Waals forces or lipophilic interactions. Liposomes and micelles are the most well known examples of these types of nanoparticles (Figure 2). These are molecules formed by noncovalent binding of their components which can self-assemble spontaneously

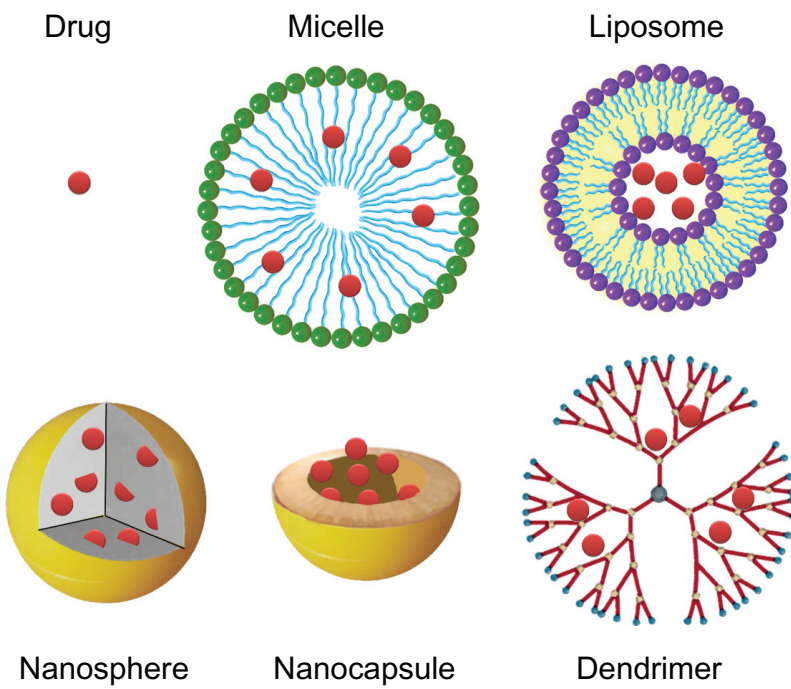

Figure 2 Schematic representation of different particulate systems for drug transport and delivery.

Notes: Some of these systems include self-assembling molecules, such as liposomes and micelles, while others are based on nonreversible organic or inorganic structures, such as nanospheres, nanocapsules, and dendrimers. and reversibly into organized structures under specific environmental conditions, eg, temperature, $\mathrm{pH}$, and polarity of the medium. ${ }^{15}$ Changes in environmental conditions usually result in disaggregation of the molecular units that form the particle. This may represent an advantage, eg, enabling release of a drug load in specific environments, such as a sudden drop in tissue $\mathrm{pH}$, as occurs in ischemic brain tissue after lactic acidosis, or be a disadvantage, eg, the molecule may disintegrate before reaching its target. Disaggregation of the nanoparticle components may be reversed by returning to the original conditions that favor their self-aggregation. Reversible nanoparticles are extremely versatile and malleable structures, easy to prepare in nonspecialized laboratories, and are the basis of preparation of new pharmaceutical approaches to the treatment of CNS disorders in neuroscience research laboratories. However, the unstable nature of these systems makes them less suitable for preparation of stable commercial products by the pharmaceutical industry. ${ }^{16}$

Conversely, the broad family of nonreversible nanoparticles (including dendrimers, nanocapsules, nanospheres, nanocages, and nanotubes, Figure 2) comprises molecules with strong molecular interactions, eg, covalent or metallic bonds, which confer a high degree of stability, thereby facilitating their manufacturing for commercial purposes, but are more rigid in their synthesis and handling. The preparation of these types of particles is less common in neuroscience research laboratories unless there is collaboration with specialized material science laboratories (a powerful coalition that is becoming quite common).

It is beyond the scope of this review to describe these structures or analyze the advantages and disadvantages of each of these molecules further, and the reader is referred to an excellent review by Vlieghe and Khrestchatisky ${ }^{13}$ for this purpose. Instead, we will focus on the use of these molecules to target the CNS.

At the dawn of the second millennium, advances achieved in the field of nanotechnology enabled us to build complex functionalized macromolecules that not only have optimized loading and release characteristics to carry and deliver therapeutic agents to the CNS in a controlled manner but also confer stealth capabilities to increase circulation time in the bloodstream, avoiding agglutination of the agent with plasmatic proteins or its retention in the liver, spleen, or other nonpathological organs, such as the lungs, enable targeting of specific tissues or cells, and include imaging probes for in vivo follow-up of these processes. The most exciting advance in recent years has been the possibility 
of performing all these tasks under the same molecular platform, which together are known as "theranostics".

\section{Liposomes and nanotechnology in theranostics: "veni, vidi, vici"}

Theranostics is a relatively new discipline in the context of personalized medicine, and involves use of nanotechnology to assemble molecular platforms that simultaneously perform a therapeutic and diagnostic function. ${ }^{17}$ Alternatively, we can define theranostics in reference to the famous Latin quotation "veni, vidi, vici" (meaning "I came, I saw, I conquered") from Julius Caesar in the year $47 \mathrm{BC}$, when commenting on his victorious campaign against Pharnaces II of Pontus. In this section, we describe how nanotechnology is essential to achieve the main goal of theranostics, ie, "to come, to see, and to conquer" disease.

\section{"Veni": theranostic agents for targeted delivery}

The most important characteristic of a theranostic agent is its ability to target the site of pathology. Most neurological disorders are focal and only affect a particular organ or tissue, eg, brain, spine, or peripheral nerves, or a region within the tissue (Figure 3A). Sometimes only specific cell types, eg, dopaminergic neurons of the substantia nigra in Parkinson's disease, or cells in a particular functional state, eg, apoptotic cells in the post-ischemic brain, are affected. Thus, systemic administration of treatment involves delivery of a drug throughout the body, in the hope that a sufficient fraction of the drug will reach the region/tissue where it is required (Figure 3B).

Systemic administration of drugs is inefficient, in that most of the drug ends up in a place where it is not required or is excreted, and is often accompanied by harmful side effects in nonpathological tissues, eg, several glutamate receptor agonists have shown a neuroprotective effect against ischemia in the laboratory but are not effective for the treatment of stroke in humans because of unacceptable side effects. Thus, it is convenient to identify strategies that facilitate concentration of active principles at the site of the disease, minimizing their presence in other tissues or regions of the body where they are not needed. This concept is known as targeted drug delivery (Figure 3C).

Theranostic agents are molecular platforms (such as liposomes, micelles, dendrimers, and nanospheres) that can be used as drug carriers to solubilize, stabilize, protect, and ultimately deliver therapeutic drugs in a controlled manner. These carriers may include "molecular antennae" such as antibodies and aptamers on their surface which allow them to interact specifically with target cells via molecular recognition mechanisms (Figure 4).

Indeed, cells that express specific molecular markers on their membranes can be used to specify their origin, phenotype, and functional status, ie, using immunohistological studies, blotting, or flow cytometry techniques. Immunovectorization of macromolecules against selected biomarkers enables "identification" of target cells and delivery of therapeutic agents only to targeted areas (Figure 4$).^{18}$

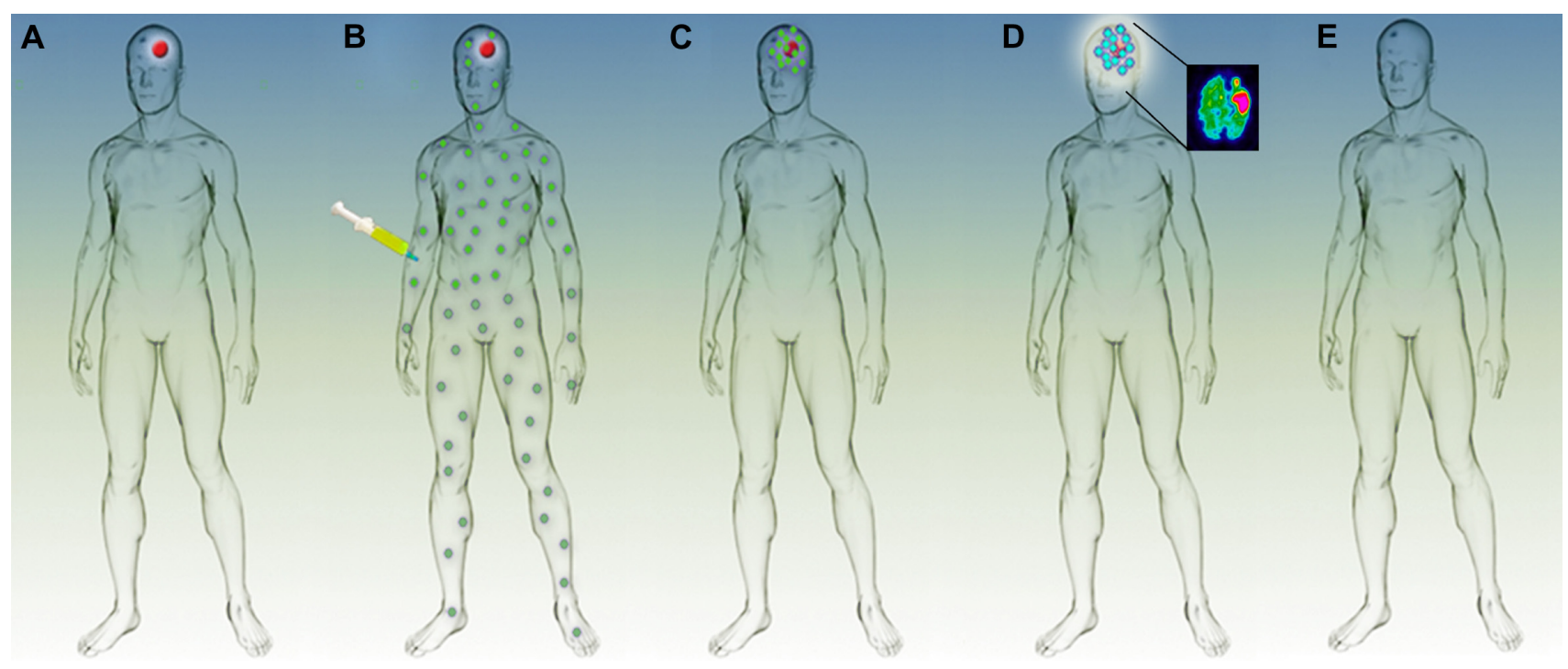

Figure 3 Concept of theranostics. (A) A pathological process localized to the brain. (B) Systemic administration of a therapeutic agent distributes the theranostic throughout the entire body. (C) Nanotechnology enables concentration of the agent in the targeted area. (D) Inclusion of imaging probes within the agent enables monitoring of the process in vivo. (E) By focusing the action of the therapeutic agent in the targeted area, the treatment becomes more effective. 

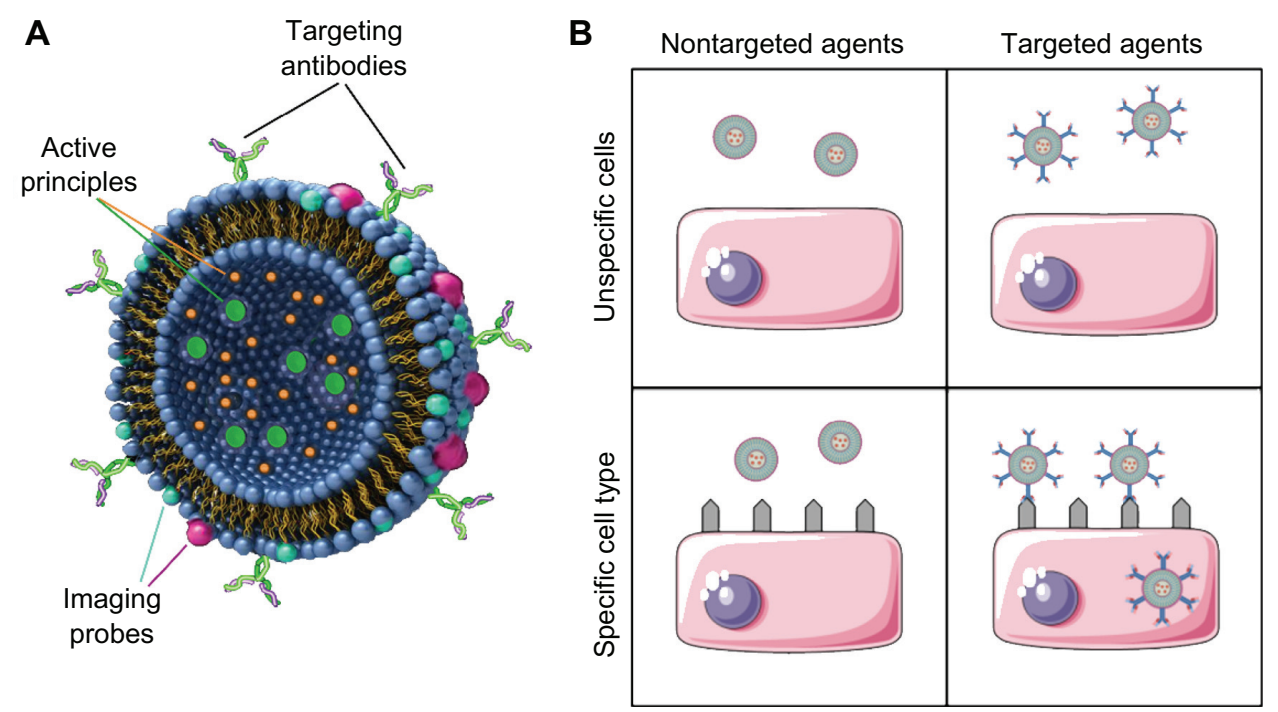

Figure 4 Theranostic agents in molecular recognition processes. (A) A liposomal theranostic agent includes surface antibodies that participate in the molecular recognition process with targeted cells, imaging probes (for diagnostic purposes), and active principles of treatment. (B) Targeting of specific cells occurs via expression of specific surface receptors against which theranostic agents are "immunized".

Note: Both immunized agents and expression of cell biomarkers (low-right corner) are required for the molecular recognition process.

A systematic and indepth review of the approaches available for crossing the blood-brain barrier is beyond the scope of this work, and the reader is referred to a review by Vlieghe and Khrestchatisky ${ }^{13}$ for this purpose.

\section{"Vidi": theranostic molecules for diagnosis}

A second requirement for a theranostic agent is inclusion of imaging probes on its surface, enabling its detection using different imaging technologies (Figure 4). In this way, the presence of the theranostic (and ultimately therapeutic) agent in the targeted area is ensured, and visualization of its spatiotemporal dynamic accumulation in the areas required is possible. The presence of imaging probes also facilitates the location, delineation, and quantification of affected tissues (ie, theranostic agents have a diagnostic function) and enables monitoring of progression of the pathological process in response to treatment on an individual basis (Figure 3D), which is a fundamental principle of personalized medicine.

In this context, and as a result of the macromolecular nature of theranostic agents, it is possible to include more than one type of imaging probe using multiple imaging techniques. Thus, it is common for theranostic agents to contain iron oxide particles or gadolinium chelates for their in vivo detection using magnetic resonance imaging, along with radioactive isotopes for detection using positron emission tomography or single photon emission computed tomography, and fluorescence probes, quantum dots, or bioluminescent probes for detection using fluorescence or optical imaging techniques. Multimodal imaging probes enable maximal exploitation of the advantages of each of these techniques, ie, the sensitivity and specificity of positron emission tomography, the spatial and temporal resolution of magnetic resonance imaging, and the microscopic resolution of fluorescence microscopy. Figure 5 shows a T1-weighted magnetic resonance image of a rat brain (at 9.4 Tesla) on the left side which was acquired one hour after an intraparenchymal injection of gadolinium-doped liposomes (their composition has been described elsewhere) ${ }^{19}$ and produced a hyperintense signal via reduction of $\mathrm{T} 1$ relaxation time in the tissue. On the right is a fluorescence microscopic image of brain tissue from the same animal, showing red fluorescence in the cytoplasm of some cells (liposomes contain rhodamine). The blue spots indicate cell nuclei in the

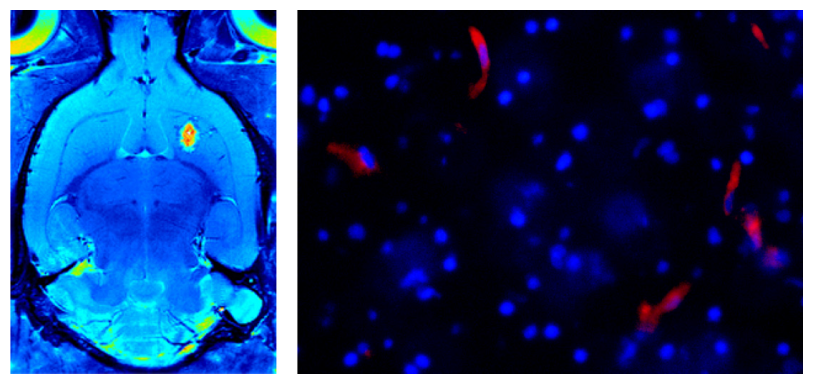

Figure 5 Multimodal imaging of a theranostic agent.

Notes: A color-coded in vivo magnetic resonance image of a rat brain, where the agent is indicated as a hyperintense area (shown on the left). An ex vivo fluorescence microscopic image of the brain tissue of the animal, indicating presence of the theranostic agent (red fluorescence) in the cytoplasm of some neurons but not in all (cellular nuclei are stained in blue), is shown on the right. (Images were obtained at our laboratories in Santiago de Compostela). 
tissue. Follow-up of the delivery process and the response of the targeted tissue to therapy enable dose modulation or treatment changes in nonresponders.

\section{"Vici": theranostic molecules to treat disease}

The ultimate role of a theranostic agent is to carry a drug, or a combination of drugs, to perform a therapeutic function and "conquer" a disease. As previously discussed, theranostic agents are advanced drug carriers and as such their structure is designed to contain an adequate load of a therapeutic agent to stabilize, transport, and release the drug in a controlled manner at its intended site of action. Several exciting examples of theranostic systems have now been reported in the literature in the treatment of cancer, ${ }^{20}$ atherosclerosis, ${ }^{21}$ and gene delivery. ${ }^{22}$

Thus far, most of the reported applications for theranostics have involved the activity of macromolecular agents at a vascular level, eg, atherosclerosis, or in tissues with a high density of blood vessels, which usually have increased permeability, eg, tumors. There is little evidence of theranostic approaches being used to target areas located inside the brain parenchyma because of the difficulties faced by systemically administered substances in crossing the blood-brain barrier. ${ }^{23}$ The multitasking nature of theranostic agents involves construction of complex nanostructures, which affect the capacity of more basic structures, ie, the drug carriers on which they are based, to cross the blood-brain barrier.
Increased size and changes in the charge and polarity of nanostructures are a more common cause of reduced blood-brain barrier permeability for these nanoplatforms, with respect to their simplified drug carriers. However, our research group and others have demonstrated that encapsulation of therapeutic agents in targeted theranostic molecules (for example, heat shock protein [HSP]72targeted liposomes) $)^{23}$ results in increased efficacy in the treatment of neurological disorders such as stroke (Figure 6). The top row of Figure 6 shows a series of T1-weighted magnetic resonance images (at 9.4 Tesla) of the brains of three rats that were subjected to an experimental model of ischemia, ${ }^{19}$ with superimposed colored maps of changes in longitudinal relaxivity (R1) 24 hours after intravenous injection of a therapeutic agent, cytidine diphosphate-choline (citicoline), in its free state (left), encapsulated in gadolinium-doped liposomes (center, composition described elsewhere by Ramos-Cabrer et al), ${ }^{19}$ or encapsulated in the same liposomes with anti-HSP72 antibodies attached to its surface to vectorize the liposomes to the peri-infarct region (right). ${ }^{23}$ Localization of the liposomes is possible because of their gadolinium load, given that gadolinium is a contrast agent that increases $\mathrm{R} 1$ relaxivity. The bottom row of the image shows pseudocolored magnetic resonance maps of transverse relaxation times (T2) for the same animals 7 days after treatment. The severity of the lesion (hyperintense on these maps) is clearly reduced by the targeted treatments.
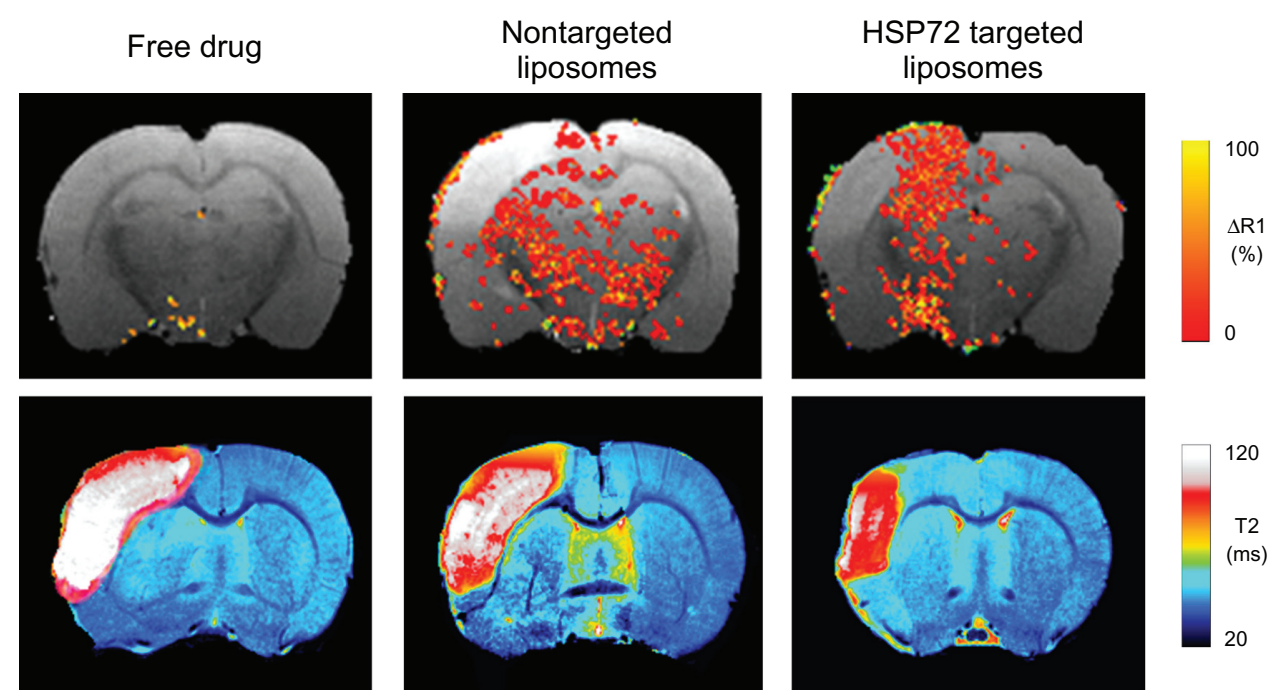

Figure 6 Theranostic activity of an agent that targets the peri-infarct region in ischemic animals.

Notes: Top row: TI magnetic resonance images with superimposed colored maps of changes in relaxivity (RI) after intravenous injection of a therapeutic agent (citicoline) in its free state (left), encapsulated in regular liposomes (center), and in heat shock protein (HSP) 72-targeted liposomes (right). Localization of the liposomes is possible because of their load of gadolinium, a contrast agent that increases RI relaxivity. Bottom row: pseudocolored magnetic resonance maps of transverse relaxation times (T2) in the same animals 7 days after treatment. The severity of the lesion is clearly reduced by targeted treatment. (Experiments performed in and magnetic resonance image obtained at our laboratories in Santiago de Compostela). 
Despite the challenges inherent in crossing the bloodbrain barrier, theranostics and nanotechnology are now providing exciting opportunities for development of novel treatments for neurological diseases. A good example of the powerful combination of both disciplines in this situation is the use of citicoline for the treatment of ischemic stroke. Our group $^{19}$ and others ${ }^{24-29}$ have demonstrated that inclusion of citicoline in liposomes results in a considerable increase (by more than 10-fold) in the bioavailability of the drug in the brain parenchyma compared with the drug administered in its free form. Thus, the known therapeutic effect of this drug in the treatment of stroke is enhanced by its encapsulation in liposomes. ${ }^{1924,25}$

Recent clinical trials have shown that citicoline is not effective for the treatment of stroke $\mathrm{e}^{31-34}$ or traumatic brain injury ${ }^{35}$ when administered orally to patients. However, the conclusions of these trials may be flawed because citicoline delivered orally may simply not reach the target brain tissue in sufficient concentrations to have beneficial effects. It is also possible that liposomal formulations of citicoline enable delivery of more intact drug into the brain, where it is able to have a therapeutic effect. Although a future clinical trial with liposome-encapsulated citicoline may offer a second opportunity for this drug, performing such a study represents a huge challenge, requiring adequate resolution of practical questions, such as how to manufacture a stable and affordable liposomal formulation for use in the research setting.

Multiple concerns will need to be overcome before engineered nanomaterials for targeted drug delivery can become a reality in everyday clinical practice. Issues such as large-scale production, cost-effectiveness, and the potential toxicity of new nanomaterials are hot topics in current state-of-the-art theranostics.

In particular, concerns regarding the potential interactions of nanostructures with biological systems have given rise to an emerging subdiscipline of nanotechnology known as "nanotoxicology", which focuses on the potential relationships between the physical and chemical properties of nanostructures (such as size, shape, surface chemistry, composition, and aggregation) and induction of toxic biological responses in vivo. An indepth review of issues such as the interaction between nanostructures and biological systems, or the biodistribution, clearance, immune response, and metabolism of nanoparticles in vivo is beyond the scope of this paper, and the reader is referred to a good review by Fischer and Chan for further details on this subject. ${ }^{36}$

\section{CNS disorders and targets: "to boldly go where no man has gone before"}

Neurological diseases have different origins and evolutions, so nanotechnology is unable to propose a universal solution for their treatment, although there are suitable solutions available for each circumstance. Some diseases are localized to a specific area that can be delineated, eg, some brain tumors, whereas others are diffuse and less specific. Some neurological diseases require chronic treatment, while others require acute intervention. A different approach may even be necessary for the acute and chronic phases of the same disease. Under these circumstances, targeting strategies and selected technological approaches used to address them need to be chosen appropriately. In general, there are three potential targeting strategies that should be considered, depending on the nature of the neurological disorder.

\section{Focal neurological disorders}

Brain tumors, traumatic brain injury, hemorrhage, and ischemic stroke are common neurological disorders where the therapeutic target is a specific area of the encephalic mass. Sometimes the therapeutic target is the pathological tissue per se (as with malignancy), but it may also be located in the surrounding areas (eg, the peri-infarct region in ischemic stroke). ${ }^{23,37}$ In either case, there are two possible strategies for targeting the site of pathology. The first strategy involves magnetic vectorization of the therapeutic agent and its retention in the area of pathology by magnetic force to increase the residence time of the agent in the brain capillaries and to facilitate their incorporation into the brain parenchyma via the blood-brain barrier. Although this concept is not new (it was initially proposed in the 1970 s by Widder et al), ${ }^{38}$ it has been used only rarely thus far. With current advances in the field of biocompatible nanomaterials, this concept has become interesting as a strategy for targeting the brain. Indeed, drug carriers, theranostic molecules, or even cells themselves may be doped with small or ultrasmall iron oxide particles that can be tolerated by the host organism and confer magnetic properties to the therapeutic agent. ${ }^{39}$ Such a concept has been exploited previously in the treatment of tumors ${ }^{40}$ and for the delivery of gene therapy. ${ }^{41}$ Moreover, in vitro evidence of their feasibility in the treatment of neurological disease has been reported ${ }^{42}$ although additional in vivo evidence is still needed to assess the true potential of magnetic drug delivery techniques in this context.

An alternative to magnetic vectorization is the use of immunonanoparticles which can also be used to accumulate 
therapeutic agents in pathological areas. In this case, it is imperative to find appropriate molecular markers for the targeted tissue to immunovectorize the therapeutic agent. For example, proteomic studies performed in our laboratories have demonstrated that neurons in the peri-infarct region (a key tissue in neuroprotective and neurorepair strategies) overexpress HSP72 in cerebral ischemia. ${ }^{23}$ After preparation, we confirmed an increased therapeutic effect of citicoline when it was encapsulated in anti-HSP72 immunoliposomes compared with its encapsulation in nonvectorized liposomes (Figure 6). In this case, accumulation of the theranostic agent in the peri-infarct area was achieved in a passive manner (whereas magnetic vectorization is an active procedure) using molecular recognition between the biomarker overexpressed by neurons in the target area (HSP72 protein) and the immunovectorized theranostic agent. However, there is no reason why both concepts cannot be combined, and future work should consider this approach.

\section{Diffuse neurological disorders}

Neurodegenerative diseases such as Parkinson's disease, Alzheimer's disease, or Huntington's disease, usually involve progressive degeneration and death of small niches of neurons throughout the encephalic mass, rendering these disorders diffuse and difficult to localize. In this case, immunovectorization of therapeutic agents against specific biomarkers of degenerating cells appears to be a more suitable approach than magnetic vectorization. A large amount of work has been done to identify appropriate biomarkers for early diagnosis of those diseases, but such research usually involves screening of blood and cerebrospinal fluid to identify target proteins involved in the development of easy-to-handle diagnostic tools in the clinical setting. ${ }^{43,44}$ Most advances in the screening of molecular biomarkers for cells inside the brain parenchyma have stemmed from the field of molecular imaging. Thus, molecules that specifically interact with pathological cells in Alzheimer's disease, ${ }^{45}$ Parkinson's disease ${ }^{46}$ and other neurodegenerative diseases have already been described in the literature. Because theranostics is considered a natural marriage of molecular imaging and drug delivery technologies, the same targets described for imaging technologies are also potentially suitable for therapeutic purposes.

\section{Whole brain disorders}

Encephalopathies are a clear example of neurological disorders that affect the entire encephalic mass without specific localized foci, for which the search for immunovectorization targeting of therapeutic/theranostic agents may be futile. Thus, more attention should be focused on a strategy for boosting access of therapeutic agents to the brain.

Inflammation and activation of the immune system is a common process in most neurological disorders. Local inflammatory reactions in the brain are characterized by an initial increase of blood flow to injured sites and selective accumulation of different effector cells from the peripheral blood. Such cells, mostly circulating neutrophils, monocytes, and locally resident macrophages, together mount a rapid inflammatory response characterized by a number of features, in particular production of cytokines. Cytokines released into the bloodstream stimulate production of chemoattractant proteins in the microglia, which drives subsequent infiltration of monocytes into the brain.

Thus, in inflammatory processes, the luminal surface of the endothelial cell expresses upregulated selectins and adhesion molecules that facilitate adhesion of leukocytes via corresponding integrins expressed on their surfaces, ${ }^{47}$ a process used by cell-mimicking nanoparticles to obtain access to the brain.

Given the ability of a number of molecules to attach to the surface of theranostic agents, one could attach integrins (similar to those expressed by monocytes) to the surfaces of nanoparticles to facilitate their incorporation into the brain via the blood-brain barrier, exploiting a mechanism similar to that used by inflammatory leukocytes. Candidate integrins for performing this task include LFA-1 (CD11a/CD18), ${ }^{48}$ Mac-1 (CD11b/CD18), and VLA-4 (CD49d/29) as a model of "synthetic leukocytes".

\section{Conclusion}

Successful experimental design of a drug treatment for neurological disease does not necessarily translate into successful therapy in the clinic. The effectiveness of the blood-brain barrier is a serious challenge in the delivery of therapeutic agents to the brain. A combination of knowledge acquired in the fields of drug delivery and molecular imaging using common molecular platforms has given rise to theranostics, a nanotechnology-based discipline that is allowing us to develop efficient tools via which drugs can cross the blood-brain barrier and reach their therapeutic targets within the brain. Using magnetic targeting or immunotargeting of cells and tissues, therapies may be made more efficient by focusing their activity in pathological tissues and by reducing unnecessary delivery of excessive amounts of drugs into the bloodstream. Further, the ability to visualize the therapeutic process in vivo will 
allow adaptation of therapies to the unique characteristics of each subject, supporting the concept of personalized medicine, which will revolutionize the treatment of patients in the coming decades.

\section{Acknowledgments}

The financial support of the Instituto Carlos III of the Spanish Ministry of Health (projects PI11/02161, CP09/00074), the Spanish Ministry of Economy and Competence (projects SAF2008-02190, SAF2011-30517, RETICS-RENEVAS RD06/0026) and the European Union (FEDER: Fondo Europeo de Desarrollo Regional program) is gratefully acknowledged. PRC acknowledges the Instituto Carlos III for a research contract as part of the Miguel Servet program.

\section{Disclosure}

The authors report no conflicts of interest in this work.

\section{References}

1. Hofman A, van Duijn CM, Franco OH, et al. The Rotterdam Study: 2012 objectives and design update. Eur J Epidemiol. 2011;26:657-686.

2. Greenhalgh AD, Ogungbenro K, Rothwell NJ, Galea JP. Translational pharmacokinetics: challenges of an emerging approach to drug development in stroke. Expert Opin Drug Metab Toxicol. 2011;7:681-695.

3. Abbott NJ, Patabendige AA, Dolman DE, Yusof SR, Begley DJ. Structure and function of the blood-brain barrier. Neurobiol Dis. 2010;37:13-25.

4. Ballabh P, Braun A, Nedergaard M. The blood-brain barrier: an overview. Structure, regulation, and clinical implications. Neurobiol Dis. 2004;16:1-13.

5. Schinkel AH. P-glycoprotein, a gatekeeper in the blood-brain barrier. Adv Drug Deliv Rev. 1999;36:179-194.

6. Abbott NJ, Romero IA. Transporting therapeutics across the blood-brain barrier. Mol Med Today. 1996;2:106-113.

7. Dhuria SV, Hanson LR, Frey WH 2nd. Intranasal delivery to the central nervous system: mechanisms and experimental considerations. J Pharm Sci. 2010;99:1654-1673.

8. Gabathuler R. Approaches to transport therapeutic drugs across the blood-brain barrier to treat brain diseases. Neurobiol Dis. 2010;37:48-57.

9. Borlongan CV, Emerich DF. Facilitation of drug entry into the CNS via transient permeation of blood brain barrier: laboratory and preliminary clinical evidence from bradykinin receptor agonist, Cereport. Brain Res Bull. 2003;60:297-306.

10. Meairs S, Alonso A. Ultrasound, microbubbles and the blood-brain barrier. Prog Biophys Mol Biol. 2007;93:354-362.

11. Suzuki M, Iwasaki Y, Yamamoto T, Konno H, Kudo H. Sequelae of the osmotic blood-brain barrier opening in rats. J Neurosurg. 1988;69: 421-428.

12. Diringer MN, Zazulia AR. Osmotic therapy: fact and fiction. Neurocrit Care. 2004;1:219-233.

13. Vlieghe P, Khrestchatisky M. Medicinal chemistry based approaches and nanotechnology-based systems to improve CNS drug targeting and delivery. Med Res Rev. March 20, 2012. [Epub ahead of print.]

14. Rautio J, Laine K, Gynther M, Savolainen J. Prodrug approaches for CNS delivery. AAPS J. 2008;10:92-102.

15. Bitounis D, Fanciullino R, Iliadis A, Ciccolini J. Optimizing druggability through liposomal formulations: new approaches to an old concept. ISRN Pharm. February 9, 2012. Article 738432.
16. Wagner A, Vorauer-Uhl K. Liposome technology for industrial purposes. Journal of Drug Delivery. 2011;2011:Article ID 591325.

17. Wang LS, Chuang MC, Ho JA. Nanotheranostics - a review of recent publications. Int J Nanomedicine. 2012;7:4679-4695.

18. Mulder WJ, Strijkers GJ, van Tilborg GA, Griffioen AW, Nicolay K. Lipid-based nanoparticles for contrast-enhanced MRI and molecular imaging. NMR Biomed. 2006;19:142-164.

19. Ramos-Cabrer P, Agulla J, Argibay B, Pérez-Mato M, Castillo J. Serial MRI study of the enhanced therapeutic effects of liposome-encapsulated citicoline in cerebral ischemia. Int J Pharm. 2011;205:228-233.

20. Fernandez-Fernandez A, Manchanda R, McGoron AJ. Theranostic applications of nanomaterials in cancer: drug delivery, image-guided therapy, and multifunctional platforms. Appl Biochem Biotechnol. 2011;165:1628-1651.

21. Lobatto ME, Fuster V, Fayad ZA, Mulder WJM. Perspectives and opportunities for nanomedicine in the management of atherosclerosis. Nat Rev Drug Discov. 2011;10:835-852.

22. Shim MS, Kwon YJ. Stimuli-responsive polymers and nanomaterials for gene delivery and imaging applications. Adv Drug Deliv Rev. 2012;64: 1046-1059.

23. Ramos-Cabrer P, Campos F, Sobrino T, Castillo J. Targeting the ischemic penumbra. Stroke. 2011;42(Suppl 1):S7-S11.

24. Adibhatla RM, Hatcher JF. Cytidine 5 '-diphosphocholine (CDP-choline) in stroke and other CNS disorders. Neurochem Res. 2005;30:15-23.

25. Adibhatla RM, Hatcher JF, Tureyen K. CDP-choline liposomes provide significant reduction in infarction over free CDP-choline in stroke. Brain Res. 2005;1058:193-197.

26. Fresta M, Puglisi G. Biological effects of CDP-choline loaded long circulating liposomes on rat cerebral post-ischemic reperfusion. Int $J$ Pharm. 1996;134:89-97.

27. Fresta M, Puglisi G. Survival rate improvement in a rat ischemia model by long circulating liposomes containing cytidine-5I-diphosphate choline. Life Sci. 1997;61:1227-1235.

28. Fresta M, Puglisi G. Reduction of maturation phenomenon in cerebral ischemia with CDP-choline-loaded liposomes. Pharm Res. 1999;16: 1843-1849.

29. Fresta M, Puglisi G, Di Giacomo C, Russo A. Liposomes as in-vivo carriers for citicoline: effects on rat cerebral post-ischaemic reperfusion. J Pharm Pharmacol. 1994;46:974-981.

30. Puglisi G, Fresta M, La Rosa C, Ventura CA, Panico AM, Mazzone G. Liposomes as a potential drug carrier for citicoline (CDP-choline) and the effect of formulation conditions on encapsulation efficiency. Pharmazie. 1992;47:211-215.

31. Davalos A, Alvarez-Sabin J, Castillo J, et al. Citicoline in the treatment of acute ischaemic stroke: an international, randomised, multicentre, placebo-controlled study (ICTUS trial). Lancet. 2012;380:349-357.

32. Clark WM, Clark TD. Stroke: treatment for acute stroke - the end of the citicoline saga. Nat Rev Neurol. 2012;8:484-485.

33. Clark WM. Efficacy of citicoline as an acute stroke treatment. Expert Opin Pharmacother. 2009;10:839-846.

34. Bustamante A, Giralt D, Garcia-Bonilla L, et al. Citicoline in pre-clinical animal models of stroke: a meta-analysis shows the optimal neuroprotective profile and the missing steps for jumping into a stroke clinical trial. J Neurochem. 2012;123:217-225.

35. Zafonte RD, Bagiella E, Ansel BM, et al. Effect of citicoline on functional and cognitive status among patients with traumatic brain injury: Citicoline Brain Injury Treatment Trial (COBRIT). JAMA. 2012;308:1993-2000.

36. Fischer HC, Chan WC. Nanotoxicity: the growing need for in vivo study. Curr Opin Biotechnol. 2007;18:565-571.

37. Lo EH. A new penumbra: transitioning from injury into repair after stroke. Nat Med. 2008;14:497-500.

38. Widder KJ, Senyel AE, Scarpelli GD. Magnetic microspheres: model system of site specific drug delivery in vivo. Proc Soc Exp Biol Med. 1978;158:141-146.

39. Laurent S, Forge D, Port M, et al. Magnetic iron oxide nanoparticles: synthesis, stabilization, vectorization, physicochemical characterizations, and biological applications. Chem Rev. 2008;108:2064-2110. 
40. Mikhaylov G, Mikac U, Magaeva AA, et al. Ferri-liposomes as an MRI-visible drug-delivery system for targeting tumours and their microenvironment. Nat Nanotechnol. 2011;6:594-602.

41. Plank C, Zelphati O, Mykhaylyk O. Magnetically enhanced nucleic acid delivery. Ten years of magnetofection - progress and prospects. Adv Drug Deliv Rev. 2011;63:1300-1331.

42. Malhotra M, Tomaro-Duchesneau C, Prakash S. Synthesis of TAT peptide-tagged PEGylated chitosan nanoparticles for siRNA delivery targeting neurodegenerative diseases. Biomaterials. 2013;34:1270-1280.

43. Lista S, Faltraco F, Hampel H. Biological and methodical challenges of blood-based proteomics in the field of neurological research. Prog Neurobiol. 2013;101-102:18-34.

44. Zhang J, Keene CD, Pan C, Montine KS, Montine TJ. Proteomics of human neurodegenerative diseases. J Neuropathol Exp Neurol. 2008;67: 923-932.
45. Matsuda H, Imabayashi E. Molecular neuroimaging in Alzheimer's disease. Neuroimaging Clin N Am. 2012;22:57-65.

46. Seibyl J, Russell D, Jennings D, Marek K. Neuroimaging over the course of Parkinson's disease: From early detection of the at-risk patient to improving pharmacotherapy of later-stage disease. Semin Nucl Med. 2012;42:406-414

47. Lossinsky AS, Shivers RR. Structural pathways for macromolecular and cellular transport across the blood-brain barrier during inflammatory conditions. Histol Histopathol. 2004;19:535-564.

48. Chen X, Wong R, Khalidov I, et al. Inflamed leukocyte-mimetic nanoparticles for molecular imaging of inflammation. Biomaterials. 2011;32:7651-7661.

\section{Publish your work in this journal}

The International Journal of Nanomedicine is an international, peerreviewed journal focusing on the application of nanotechnology in diagnostics, therapeutics, and drug delivery systems throughout the biomedical field. This journal is indexed on PubMed Central, MedLine, CAS, SciSearch $\AA$, Current Contents ${ }^{\circledR} /$ Clinical Medicine,
Journal Citation Reports/Science Edition, EMBase, Scopus and the Elsevier Bibliographic databases. The manuscript management system is completely online and includes a very quick and fair peer-review system, which is all easy to use. Visit http://www.dovepress.com/ testimonials.php to read real quotes from published authors. 